\title{
Semi-Classical Asymptotics in Solid State Physics
}

\author{
J. C. Guillot ${ }^{1}$, J. Ralston ${ }^{2}$ and E. Trubowitz ${ }^{3}$ \\ 1 Universite de Paris-Nord, Villetaneuse, France \\ 2 University of California, Los Angeles, California 90024, USA \\ ${ }^{3}$ ETH, CH-8093 Zurich, Switzerland
}

\begin{abstract}
This article studies the Schrödinger equation for an electron in a lattice of ions with an external magnetic field. In a suitable physical scaling the ionic potential becomes rapidly oscillating, and one can build asymptotic solutions for the limit of zero magnetic field by multiple scale methods from "homogenization." For the time-dependent Schrödinger equation this construction yields wave packets which follow the trajectories of the "semiclassical model." For the time-independent equation one gets asymptotic eigenfunctions (or "quasimodes") for the energy levels predicted by Onsager's relation.
\end{abstract}

This article initiates a study of an approximation widely used in the quantum theory of solids. When one ignores interactions between electrons in a crystal, one is quickly led to consider the one-body Hamiltonian governing the motion of a single electron through a lattice of ions in the presence of external electric and magnetic fields. If the external fields are effectively constant in space and one is interested in wave packets which are large relative to the lattice spacing, a simplified theory for the motion of the packets, known as "the semi-classical model," has been in use since the 1930's. In particular, for the case of an external magnetic field with vector potential $A(x)$, R. Peierls [13] concluded that suitably prepared packets would follow the orbits of the classical Hamiltonian

$$
H(x, p)=E_{n}(p+A(x)),
$$

where $E_{n}(k)$ is an energy band function for the (Bloch) spectrum of the problem without the external field. Through the years there have been a number of efforts to justify this approximation and/or exhibit solutions of the Schrödinger equation with this behavior (Kohn [10], Chambers [3], Zak [14]). However, to the best of our knowledge there has not been a study using multiple-scale techniques as in homogenization. We feel that this approach simplifies the justification of the model considerably. It also makes it possible to refine the model and extend it.

In this paper we only consider problems with external magnetic fields. After discussing the physically relevant scaling of the equations, we begin by constructing 
approximate solutions to the time-dependent Schrödinger equation whose supports follow the orbits of $H(x, p)$. This construction can be carried out to all orders in $\varepsilon$, where $\varepsilon=1.5 \times 10^{-9} \times B$ and $B$ is the magnetic field strength in Gauss. Thus the "asymptotics" in our title refer to the limit $B \rightarrow 0$, not $\hbar \rightarrow \dot{0}$.

The second half of the paper is devoted to the construction of approximate eigenfunctions or "quasimodes" for the magnetic Hamiltonian. One of the most important predictions of the semi-classical model (when combined with the Bohr-Sommerfeld quantization rule) is Onsager's relation [12]. This gives a formula for energy levels of an electron in a crystal in a magnetic field, and it is the basis for calculation of Fermi surfaces using data from the de Hass-van Alphen experiment. We construct approximate eigenfunctions for the energy levels predicted by Onsager's relation. From a technical point of view this is quite easy, because the problem is essentially one-dimensional in the "slow" variables. In any case the constructions require only a rather direct combination of the Ansätze of homogenization and geometric optics with caustics. Once again the constructions can be carried out to all orders in $\varepsilon$ and here this gives a refinement of Onsager's relation.

Throughout this article the function $E_{n}(k)$ is assumed to correspond to a simple eigenvalue. In the future we hope to give semi-classical asymptotics for the time-dependent Schrödinger equation for cases where $E_{n}\left(k_{0}\right)=E_{n+1}\left(k_{0}\right)$, and the function $E_{n}(k)$ has a conical singularity at $k_{0}$. This occurs in graphite.

Finally we wish to call the reader's attention to the recent work of Helffer and Sjostrand on semi-classical asymptotics for Schrödinger's equation with magnetic fields, [6] and [7]. This treats a variety of problems which differ from those considered here in that the ionic potential is not rapidly oscillating on the scale which makes the coefficients of the differentiations small.

\section{Scaling}

The Hamiltonian for an electron in a crystal lattice in the presence of a constant external magnetic field of strength $B$ and direction $\omega$ is given by

$$
H=\frac{1}{2 m}\left(i \hbar \frac{\partial}{\partial x}+\frac{e B}{2 c} \omega \times x\right)^{2}+V(x) .
$$

Here $m$ and $e$ are the electron mass and charge respectively, $c$ is the speed of light and the potential $V$ is assumed to be smooth and periodic on the crystal lattice. The spacing in typical crystal lattices is on the order of Angstroms and typical ionization potentials are the order of electron volts. Hence, $V$ and its derivatives are of order one in these units, which makes them suitable for the problem at hand. In these units

$$
H=\alpha\left(i \frac{\partial}{\partial x}+\frac{\beta}{2} B \omega \times x\right)^{2}+V(x),
$$

where $\alpha=3.81 \mathrm{eV}(\AA)^{2}$ and $\beta=1.52 \times 10^{-9}(\AA)^{-2} /$ Gauss. Since experimental field strengths do not usually exceed the order of $10^{5}$ Gauss, we will treat $\varepsilon=\beta B$ as a small parameter in what follows. 
The choices of distance and energy variables made here were determined by the need to make $V(x)$ and its derivatives of order one. This has lead us to something close to "atomic units" $(\hbar=m=e=1)$. Indeed, in what follows we will move closer to that system and suppress $\alpha$. The constant $\alpha$ is of order one, and removing it corresponds to a minor change in energy units. Considerations of this type do not fix a time variable. We will be interested in wave packets whose spatial dimensions are order one in the variable $y=\varepsilon x \sim 10^{-5} x$, and we will see that these packets can be expected to persist for times that are order one in the variable $s=\varepsilon \hbar^{-1} t \sim 10^{10} t$, where $t$ is in seconds. Rewriting the Schrödinger equation in these variables, we have

$$
i \varepsilon \frac{\partial u}{\partial s}=\left(i \varepsilon \frac{\partial}{\partial y}+\frac{\omega \times y}{2}\right)^{2} u+V\left(\frac{y}{\varepsilon}\right) u .
$$

This is the form of the equation that we will use from here on, and as noted above, we will treat $\varepsilon$ as a parameter that can be as small as we wish.

\section{Time-Dependent Wave Packets}

For equations like (1) Ansätze for asymptotic solutions (in the limit $\varepsilon \rightarrow 0$ ) are available. In particular, following Chap. V of Benssousan, Lions and Papanicolaou [2], we look for $u$ in the form

$$
u(y, s, \varepsilon)=e^{(-i / \varepsilon) \varphi(y, s)} m\left(\frac{y}{\varepsilon}, y, \varepsilon\right),
$$

where $m(x, y, s, \varepsilon)=m_{0}(x, y, s)+\varepsilon m_{1}(x, y, s)+\cdots$ and $m$ is assumed to have the periodicity of $V$ in $x$. Making this substitution we have

$$
i \varepsilon \frac{\partial u}{\partial s}-H u=e^{(-i / \varepsilon) \varphi}\left(L_{0} m+\varepsilon L_{1} m+\varepsilon^{2} L_{2} m\right)
$$

where

$$
\begin{aligned}
& L_{0}=\frac{\partial \varphi}{\partial s}(y, s)-\left(i \frac{\partial}{\partial x}+k\right)^{2}-V(x), \\
& L_{1}=i \frac{\partial}{\partial s}-2 i k \cdot \frac{\partial}{\partial y}+2 \frac{\partial}{\partial x} \cdot \frac{\partial}{\partial y}-i \frac{\partial}{\partial y} \cdot k,
\end{aligned}
$$

and $L_{2}=\partial / \partial y \cdot \partial / \partial y$. Here the vector $k$ is given by

$$
k(y, s)=\frac{\partial \varphi}{\partial y}+\frac{\omega \times y}{2} .
$$

Thus to solve the Schrödinger equation to order $\varepsilon^{2}$ we will need

$$
\begin{aligned}
& L_{0} m_{0}=0 \quad \text { and } \\
& L_{0} m_{1}=-L_{1} m_{0} .
\end{aligned}
$$


Equation (3.1) says that for all $(y, s) m_{0}(x, y, s)$ is an eigenfunction of

$$
H_{0}=\left(i \frac{\partial}{\partial x}+k\right)^{2}+V(x)
$$

with eigenvalue $\partial \varphi / \partial s$, satisfying the periodicity conditions in $x$. Following the usual conventions for Bloch spectrum, we let $E_{n}(k), k \in \mathbf{R}^{3}$, be the $n^{\text {th }}$ largest eigenvalue of $H_{0}(k)$ with the periodicity conditions, and $\psi_{n}(x, k)$ be the corresponding eigenfunction, chosen so that the sequence $\left\{\psi_{n}\right\}_{n=1}^{\infty}$ is orthonormal in $L^{2}(D), D$ a fundamental domain for the period lattice. Hence, assuming that $E_{n}(k)$ is a simple eigenvalue when $k$ is given by (2), we can satisfy (3.1) by choosing $\varphi$ so that

$$
\frac{\partial \varphi}{\partial s}=E_{n}\left(\frac{\partial \varphi}{\partial y}+\frac{\omega \times y}{2}\right)
$$

and setting

$$
m_{0}=f_{0}(y, s) \psi_{n}\left(x, \frac{\partial \varphi}{\partial y}+\frac{\omega \times y}{2}\right) .
$$

In view of (4.1) it follows by the Fredholm alternative that (3.2) can be solved for $m_{1}$ if and only if

$$
0=\int_{D} \bar{\psi}_{n}\left(x, \frac{\partial \varphi}{\partial y}+\frac{\omega \times y}{2}\right) L_{1} m_{0} d x
$$

After some computation (see Appendix 1), (5) reduces to

$$
\frac{\partial f_{0}}{\partial s}=\frac{\partial E_{n}}{\partial k}\left(\frac{\partial \varphi}{\partial y}+\frac{\omega \times y}{2}\right) \cdot \frac{\partial f_{0}}{\partial y}+\frac{1}{2}\left(\frac{\partial}{\partial y} \cdot \frac{\partial E_{n}}{\partial k}\left(\frac{\partial \varphi}{\partial y}+\frac{\omega \times y}{2}\right)\right) f_{0}+i b f_{0},
$$

where $b$ is the (real-valued) function

$$
b=\left.2 i \int_{D}\left(i \frac{\partial}{\partial x}+k\right) \psi_{n} \cdot A \frac{\partial}{\partial k} \bar{\psi}_{n} d x\right|_{k=(\partial \varphi / \partial y)+\omega \times y} .
$$

Here $A$ is the Jacobian of $\omega \times y$

$$
\left(\begin{array}{ccc}
0 & -w_{3} & w_{2} \\
w_{3} & 0 & -w_{1} \\
-w_{2} & w_{1} & 0
\end{array}\right) .
$$

By standard Hamilton-Jacobi theory (4.1) implies that the curves defined by

$$
\dot{y}=\frac{\partial E_{n}}{\partial k}\left(\frac{\partial \varphi}{\partial y}+\frac{\omega \times y}{2}\right)
$$

must be the spatial parts of solutions to

$$
\begin{aligned}
& \dot{y}=\frac{\partial E_{n}}{\partial k}\left(\xi+\frac{\omega \times y}{2}\right), \\
& \dot{\xi}=-\frac{\partial}{\partial y}\left(E_{n}\left(\xi+\frac{\omega \times y}{2}\right)\right)=\frac{\omega}{2} \times \frac{\partial E_{n}}{\partial k}\left(\xi+\frac{\omega \times y}{2}\right) .
\end{aligned}
$$


Note that in terms of $k=\xi+(\omega \times y) / 2$ this system is the semi-classical model ([1]),

$$
\dot{y}=\frac{\partial E_{n}}{\partial k}(k) \quad \dot{k}=\omega \times \frac{\partial E_{n}}{\partial k}(k) .
$$

Thus the transport equation (6) implies that if we begin with a localized packet, i.e. choose $f(y, 0)$ with small support, the packet will move along the trajectory predicted by the semi-classical theory. The coefficient

$$
\frac{1}{2}\left(\frac{\partial}{\partial y} \cdot \frac{\partial E_{n}}{\partial k}\left(\frac{\partial \varphi}{\partial y}+\frac{\omega \times y}{2}\right)\right)
$$

insures that $\int_{\mathbf{R}^{3}}|u|^{2} d y$ is constant in time modulo terms of order $\varepsilon$. We do not know the physical significance of $b$, but it contributes a variation in the phase of $u$. Mathematically $b$ plays exactly the rôle in these constructions that the "subprincipal symbol" plays in the construction of asymptotic solutions to $P(x, \varepsilon D) u=0$, cf. [4], $\S 1.3$.

We can go on to solve the Schrödinger equation to order $\varepsilon^{N}$ for any $N$. To eliminate terms of order $\varepsilon^{2}$, we must solve

$$
L_{0} m_{2}=-L_{1} m_{1}-L_{2} m_{0} .
$$

As in solving (3.2), this can be solved for $m_{2}$ if and only if

$$
0=\int_{D} \bar{\psi}_{n}\left(L_{1} m_{1}+L_{2} m_{0}\right) d x
$$

Since (3.2) only determines $m_{1}$ up to $f_{1}(y, s) \psi_{n}(x, k(y, s))$, (7) reduces to an inhomogeneous version of the transport equation (6) for $f_{1}$. The equations obtained by setting the coefficients of $\varepsilon^{N}$ to zero for $N>2$ are all solved the same way.

One we have constructed $u$ satisfying the Schrödinger equation to order $\varepsilon^{N}$, the standard argument from Duhamel's formula shows that $u(s)$ differs from the true solution of the Schrödinger equation with initial data $u(0)$ in $L^{2}$-norm by an error bounded by $C|s| \varepsilon^{N}$, where $C$ is independent of $s$ and $\varepsilon$.

\section{Quasi-Modes and Onsager's Relation}

In this section we will construct a large family of approximate eigenfunctions for the Hamiltonian

$$
H(\varepsilon)=\left(i \varepsilon \frac{\partial}{\partial y}+\frac{\omega \times y}{2}\right)^{2}+V\left(\frac{y}{\varepsilon}\right)
$$

with eigenvalues near a fixed energy $E_{0}$. When $E_{0}$ is taken to be Fermi energy, the contribution of these eigenvalues to the spectral density of $H(\varepsilon)$ will confirm Onsager's explanation of the de Haas-van Alphen effect. However, to avoid dealing with operators with continuous spectrum, we will follow the usual procedure of considering $H(\varepsilon)$ restricted to functions satisfying $u(x+d)=u(x)$ for $d$ in a sublattice of the crystal lattice $L$. To have convenient sublattices for the semi-classical constructions that follow we will assume that the points in $L$ and $\omega$ have rational coordinates in terms of an orthonormal basis for $E^{3}$. Hence we can choose an 
orthogonal basis for $E^{3}$, including a vector parallel to $\omega$, with rational coordinates in terms of a basis for $L$. Thus we have an orthonormal basis for $E^{3},\left\{\hat{e}_{i}: i=1,2,3\right\}$ such that $\hat{e}_{3}=\omega$ and $\alpha_{i} e_{i} \in L, i=1,2,3$, for some positive $\alpha_{i}$ 's -we choose the smallest ones. We will let $L_{N}$ be the sublattice of $L$ generated by $\left\{N \alpha_{i} \tilde{e}_{i}: i=1,2,3\right\}$, and consider $H(\varepsilon)$ restricted to functions satisfying $u(x+d)=u(x), d \in L_{N}$. As $N \rightarrow \infty$ the spectral densities for these problems converge to the spectral density for $H(\varepsilon)$ as an operator on $L^{2}\left(\mathbf{R}^{3}\right)$.

Taking $\left(y_{1}, y_{2}, y_{3}\right)$ as coordinates in terms of $\left\{\hat{e}_{1}, \hat{e}_{2}, \hat{e}_{3}\right\}$, we have

$$
\frac{\omega \times y}{2}=\frac{-y_{2}}{2} \hat{e}_{1}+\frac{y_{1}}{2} \hat{e}_{2}
$$

However, in $H(\varepsilon)$ we will use $y_{1} \hat{e}_{2}$ in place of $\omega \times y / 2$. This gauge transformation does not change the spectrum of $H(\varepsilon)$ as an operator on $L^{2}\left(\mathbf{R}^{3}\right)$, since it corresponds to conjugation by the operator of multiplication by $\exp \left(-i y_{1} y_{2} / 2\right)$.

Our construction will follow the method, initiated in Keller [8] and Keller-Rubinow [9] and developed in Maslov [11] and Duistermaat [4], of associating approximate eigenfunctions with Lagrangian manifolds invariant under the flow of the Hamiltonian $E_{n}\left(\xi+y_{1} \hat{e}_{2}\right)$. The oscillatory coefficient $V(y / \varepsilon)$ will only introduce minor modifications in this construction. Accordingly, we will begin with a 3-parameter family of Lagrangian manifolds on which the simple eigenvalue $E_{n}(k)$ satisfies

$$
E_{n}\left(\xi+y_{1} \hat{e}_{2}\right)-E=0 .
$$

We will assume that $\left(\partial E_{n} / \partial k_{1}, \partial E_{n} / \partial k_{2}\right)$ does not vanish on

$$
\Gamma_{0}=\left\{\left(k_{1}, k_{2}\right): E_{n}\left(k_{1}, k_{2}, k_{3}^{0}\right)=E_{0}\right\},
$$

and that a connected component of $\Gamma_{0}$ is a simple closed curve $\gamma_{0}$-recall that $E_{n}(k+\delta)=E_{n}(k)$ for all $\delta$ in the dual lattice to $L$. The nonvanishing of the partial gradient insures that

$$
\Gamma\left(E, k_{3}\right)=\left\{\left(k_{1}, k_{2}\right): E_{n}\left(k_{1}, k_{2}, k_{3}\right)=E\right\}
$$

has the same form as $\Gamma_{0}$ for $\left|E-E_{0}\right|<c$ and $\left|k_{3}-k_{3}^{0}\right|<c$, and that it depends smoothly on $\left(E, k_{3}\right)$. We let $\gamma\left(E, k_{3}\right)$ be the simple closed curve in $\Gamma\left(E, k_{3}\right)$ which converges to $\gamma_{0}$ as $\left(E, k_{3}\right)$ goes to $\left(E_{0}, k_{3}^{0}\right)$.

For $\left|k_{3}-k_{3}^{0}\right|<c$ and $\left|E-E_{0}\right|<c$ we define the (Lagrangian) manifolds

$$
L\left(E, k_{2}, k_{3}\right)=\left\{(y, \xi): \xi_{2}=k_{2}, \xi_{3}=k_{3},\left(\xi_{1}, k_{2}+y_{1}\right) \in \gamma\left(E, k_{3}\right)\right\} .
$$

Near points on $L\left(E, k_{2}, k_{3}\right)$ corresponding to points on $\gamma\left(E, k_{3}\right)$ where $\partial E_{n} / \partial k_{1} \neq 0$, we have $L\left(E, k_{2}, k_{3}\right)$ given by $(y, \partial S / \partial y)$, where $S=f\left(y_{1}+k_{2}\right)+k_{2} y_{2}+k_{3} y_{3}$ and $f$ satisfies $E_{n}\left(f^{\prime}(z), z, k_{3}\right)=E$. Near points on $L\left(E, k_{2}, k_{3}\right)$ corresponding to points on $\gamma\left(E, k_{3}\right)$ where $\partial E_{n} / \partial k_{1}=0, L\left(E, k_{2}, k_{3}\right)$ is given by $\{(y, \partial S / \partial y): \partial S / \partial \alpha=0\}$, where

$$
S=\left(y_{1}+k_{2}\right) \alpha-h(\alpha)+k_{2} y_{2}+k_{3} y_{3}
$$

and $h$ is defined by $E_{n}\left(\alpha, h^{\prime}(\alpha), k_{3}\right)=E$.

In this paragraph we will attempt to give an overview of the construction of 
approximate eigenfunctions "associated" with the family of Lagrangian manifolds $L\left(E, k_{2}, k_{3}\right)$. For readers familiar with this type of construction (we follow Sect. 1 of Duistermaat [4] closely) this sketch may suffice without the more detailed discussion that follows it. We will build approximate solutions to $H(\varepsilon) u-\left(E+\varepsilon E_{1}\right) u=0$ using the Ansätze

$$
u=e^{(-i / \varepsilon) S(y)} m(y / \varepsilon, y, \varepsilon)
$$

and

$$
u=(2 \pi \varepsilon)^{-1 / 2} \int_{\mathbf{R}} e^{(-i / \varepsilon) S(y, \alpha)} m(y / \varepsilon, y, \alpha, \varepsilon) d \alpha,
$$

where $S(y)$ and $S(y, \alpha)$ are phase functions parametrizing parts of $L\left(E, k_{2}, k_{3}\right)$ as described in the preceding paragraph. Given any $y_{1}^{0}$ we will be able to build functions satisfying the eigenvalue equation to order $\varepsilon^{2}$ near $y_{1}=y_{1}^{0}$, corresponding to all the sheets of $L\left(E, k_{2}, k_{3}\right)$ lying above a neighborhood of $y_{1}=y_{1}^{0}$. However, we will need to patch these local solutions together via a partition of unity in $y_{1}$ to give a globally defined solution. This will force us to choose the undermined additive constants in the phase functions in a consistent way and impose constraints on $\left(E, k_{2}, k_{3}\right)$. The constraints on $k_{2}$ and $k_{3}$ are simply those which make the phase functions satisfy the periodicity conditions, i.e.

$$
k_{i}=\frac{2 \pi m_{i}}{\alpha_{i} N}, \quad m_{i} \in \mathbf{Z}, \quad i=2,3,
$$

but $E$ is constrained by

$$
\int_{\tilde{\gamma}\left(E, k_{3}\right)} \xi \cdot d y=2 \pi \varepsilon\left(m+\frac{1}{2}\right), \quad m \in \mathbf{Z},
$$

where $\tilde{\gamma}$ is any curve homotopic in $L\left(E, k_{2}, k_{3}\right)$ to $L\left(E, k_{2}, k_{3}\right) \bigcap\left\{y_{2}=y_{2}^{0}, y_{3}=y_{3}^{0}\right\}$. Condition (9) is the familiar Bohr-Sommerfeld quantization condition, since the Maslov index of $\tilde{\gamma}$ is 2, because $\tilde{\gamma}$ is a simple closed curve. Using our explicit description of $L\left(E, k_{2}, k_{3}\right)$, we see that (9) is equivalent to

$$
\text { Area enclosed by } \gamma\left(E, k_{3}\right)=2 \pi \varepsilon\left(m+\frac{1}{2}\right), \quad m \in \mathbf{Z} \text {. }
$$

This is Onsager's relation. In the literature (e.g. $[1,10])$ one finds this formula with $1 / 2$ replaced by a function of $\left(E, k_{3}\right)$ which is not necessarily $1 / 2$. However, if one interprets $E$ as the leading term in an expansion of the energy levels in powers of $\varepsilon$, as we do here, $1 / 2$ is the only possible value.

Precisely as in our construction of time-dependent wave packets, the $u$ given by (8.1) will satisfy $H(\varepsilon) u-\left(E+\varepsilon E_{1}\right) u=O\left(\varepsilon^{2}\right)$, provided $m(y / \varepsilon, y, \varepsilon)$ satisfies the time-independent versions of (4.2) and (6), i.e.

$$
m_{0}(x, y)=f(y) \psi_{n}\left(x, k\left(y_{1}\right)\right),
$$

where $k\left(y_{1}\right)=\partial S / \partial y+y_{1} \hat{e}_{2}$ and

$$
0=\frac{\partial E_{n}}{\partial k}\left(k\left(y_{1}\right)\right) \cdot \frac{\partial f}{\partial y}+\frac{1}{2}\left(\frac{\partial}{\partial y} \cdot \frac{\partial E_{n}}{\partial k}\left(k\left(y_{1}\right)\right)\right) f+i\left(b+E_{1}\right) f .
$$


Since the flow

$$
\dot{y}=\frac{\partial}{\partial \xi}\left(E_{n}\left(\xi+y_{1} \hat{e}_{2}\right)\right) \quad \dot{\xi}=-\frac{\partial}{\partial y}\left(E_{n}\left(\xi+y_{1} \hat{e}_{2}\right)\right),
$$

leaves $L\left(E, k_{2}, k_{3}\right)$ invariant, it is natural to treat $y$ as a coordinate on $L\left(E, k_{2}, k_{3}\right)$ on the set where we use (8.1). Then the differentiation in (11) is just the derivative of $f$ along the flow (12). Moreover, the second term in (11) insures that the density $|f|^{2} d y$ is invariant under the flow. Finally, the coefficient $b$ in (11) is the restriction to $L\left(E, k_{2}, k_{3}\right)$ of the smooth function on $(y, \xi)$-space

$$
\left.2 i \int_{D}\left(i \frac{\partial}{\partial x}+k\right) \psi_{n} \cdot A \frac{\partial}{\partial k} \bar{\psi}_{n} d x\right|_{k=\xi+y_{1} \hat{e}_{2}},
$$

(see Appendix I.)

One sees immediately that all coefficients in (11) are functions of $y_{1}$ alone. This makes solving (11) much simpler than it is in the general case (i.e. in [4]). Since the flow in (12) commutes with translations in $y_{2}$ and $y_{3}$, there is a well-defined minimal positive $s_{0}$ such that $\left(y_{1}\left(s+s_{0}\right), \xi_{1}\left(s+s_{0}\right)\right)=\left(y_{1}(s), \xi_{1}(s)\right)$ for any orbit of (12) on $L\left(E, k_{2}, k_{3}\right)$. Taking a specific orbit, $(y(s), \xi(s))$ we set

$$
\begin{aligned}
& E_{1}=\frac{-1 \int_{0}^{s_{0}} b\left(\xi_{1}(s), k_{2}+y_{1}(s), k_{3}\right) d s \quad \text { and }}{s_{0}} \\
& \left.c(s)=\int_{0}^{s} b\left(\xi_{1}(t), k_{2}+y_{1}(t), k_{3}\right)+E_{1}\right) d t .
\end{aligned}
$$

Then, defining $d(y, \xi)=c(s)$ for all points $(y, \xi)$ of $L\left(E, k_{2}, k_{3}\right)$ with $y_{1}=y_{1}(s)$, $\xi_{1}=\xi_{1}(s)$, we get a smooth function on $L\left(E, k_{2}, k_{3}\right)$ satisfying

$$
\frac{\partial E_{n}}{\partial k}\left(\frac{\partial S}{\partial y}+y_{1} \hat{e}_{2}\right) \cdot \frac{\partial d}{\partial y}=b+E_{1}
$$

on subsets of $L\left(E, k_{2}, k_{3}\right)$ coordinatized by $y$. Note that $d$ is independent of $y_{2}$ and $y_{3}$.

Letting $f=e^{-i d} g$, Eq. (11) merely says that the density $g^{2} d y$ is invariant under the flow of (12). One such density, defined globally on $L\left(E, k_{2}, k_{3}\right)$ is " $d s d y_{2} d y_{3}$," where

$$
\int_{L\left(E, k_{2}, k_{3}\right)} h d s d y_{2} d y_{3}=\int_{\mathbf{R}^{2}} d y_{2} d y_{3} \int_{0}^{s_{0}} h\left(y_{1}(s), y_{2}, y_{3}, \xi_{1}(s), k_{2}, k_{3}\right) d s
$$

for all $h \in C_{0}^{\infty}\left(L\left(E, k_{2}, k_{3}\right)\right)$. On any open set in $L\left(E, k_{2}, k_{3}\right)$ that can be coordinatized by $y, d s d y_{2} d y_{3}=g^{2}\left(y_{1}\right) d y_{1} d y_{2} d y_{3}$, where $g$ is a smooth positive function such that $f=e^{-i d} g$ satisfies (12).

The only points of $L\left(E, k_{2}, k_{3}\right)$ which do not have neighborhoods coordinatized by $y$ are those for which

$$
\frac{\partial E_{n}}{\partial k_{1}}\left(\xi_{1}, k_{2}+y_{1}, k_{3}\right)=0
$$

Since $\gamma\left(E, k_{3}\right)$ is a real-analytic closed curve (since $E_{n}(k)$ is simple, $E_{n}(k)$ is a 
real-analytic function of $k$ ), it follows that (14) holds only for a finite set $\Sigma$ of values of $\left(y_{1}, \xi_{1}\right)$ on $L\left(E, k_{2}, k_{3}\right)$. It is near the $y_{1}$ 's corresponding to points in $\Sigma$ that we will use Ansätz (8.2). To complete the construction we only need to show:

(a) that we can satisfy $H(\varepsilon) u-\left(E+\varepsilon E_{1}\right) u=O\left(\varepsilon^{3 / 2}\right)$ using (8.2), and

(b) we can make choices so that $u$ in (8.2) agrees to $O\left(\varepsilon^{2}\right)$ away from $\Sigma$ with

$$
e^{(i / \varepsilon) S(y)} e^{-i d\left(y_{1}\right)} g\left(y_{1}\right) \psi_{n}\left(\frac{y}{\varepsilon}, \frac{\partial S}{\partial y}+y_{1} \hat{e}_{2}\right),
$$

where $d s d y_{2} d y_{3}$ in (13) is given by $g^{2} d y_{1} d y_{2} d y_{3}$.

In (8.2) we are going to take $m(x, y, \alpha, \varepsilon)=m_{0}(x, y, \alpha)+\varepsilon m_{1}(x, y, \alpha)$ with $m_{0}(x, y, \alpha)=f(y, \alpha) \psi_{n}\left(x,(\partial / \partial y) S(y, \alpha)+y_{1} \hat{e}_{2}\right)$. Then we have $H(\varepsilon) u-\left(E+\varepsilon E_{1}\right) u=$ $I_{1}+I_{2}$, where

and

$$
I_{1}=\varepsilon^{-1 / 2} \int_{\mathbf{R}} e^{(-i / \varepsilon) S}\left(E_{n}\left(k\left(y_{1}, \alpha\right)\right)-E\right) f(y, \alpha) \psi_{n}\left(y / \varepsilon, k\left(y_{1}, \alpha\right)\right) d \alpha
$$

$$
I_{2}=\varepsilon^{-1 / 2} \int_{\mathbf{R}} e^{(-i / \varepsilon) S}\left(\varepsilon L_{0} m_{1}+\varepsilon L_{1} m+\varepsilon^{2} L_{2} m\right) d \alpha,
$$

with $k\left(y_{1}, \alpha\right)=\partial S / \partial y+y_{1} \hat{e}_{2}$,

$$
\begin{aligned}
& L_{0}=(i \partial / \partial x+k)^{2}+V(x)-E, \\
& L_{1}=2 i k \cdot \partial / \partial y-2 \partial / \partial x \cdot \partial / \partial y+i \partial / \partial y \cdot k-E_{1}, \\
& L_{2}=\partial / \partial y \cdot \partial / \partial y \text { and } k=k\left(y_{1}, \alpha\right) .
\end{aligned}
$$

The first observation one needs here is that in a neighborhood of a point $\left(y^{0}, \alpha^{0}\right)$ corresponding to a point in $\Sigma$ one has

$$
E_{n}\left(\frac{\partial S}{\partial y}(y, \alpha)+y_{1} \hat{e}_{2}\right)-E=\frac{\partial S}{\partial \alpha}(y, \alpha) R(y, \alpha)
$$

where $R$ is smooth. This follows since by construction

$$
E_{n}\left(\frac{\partial S}{\partial y}+y_{1} \hat{e}_{2}\right)=E
$$

when $(\partial S / \partial \alpha)=0$, and $\left(\partial^{2} S / \partial \alpha \partial y_{1}\right) \neq 0$. Moreover, differentiating (15) with respect to $y_{1}$ and evaluating on $L\left(E, k_{2}, k_{3}\right)$ one sees

$$
\frac{\partial E_{n}}{\partial k_{2}}\left(\frac{\partial S}{\partial y}+y_{1} \hat{e}_{2}\right)=R(y, \alpha) \text {. }
$$

In view of (15), assuming $y_{1}$ is sufficiently close to $y_{1}^{0}$, we can integrate by parts in $I_{1}$. This gives

$$
I_{1}=\frac{\varepsilon^{1 / 2}}{i} \int_{\mathbf{R}} e^{(-i / \varepsilon) S} \frac{\partial}{\partial \alpha}\left(R(y, \alpha) f(y, \alpha) \psi_{n}\left(y / \varepsilon, k\left(y_{1}, \alpha\right)\right) d \alpha .\right.
$$

Thus, if we can find $m_{2}$ so that

$$
L_{0} m_{1}+L_{1} m_{0}-i \frac{\partial}{\partial \alpha}\left(R(y, \alpha) m_{0}\right)=\frac{\partial S}{\partial \alpha} R_{1}(y, \alpha) \psi_{n}\left(x, \frac{\partial S}{\partial y}+y_{1} \hat{e}_{2}\right),
$$


we will have

$$
H(\varepsilon) u-\left(E+\varepsilon E_{1}\right) u=\varepsilon^{3 / 2} \int_{\mathbf{R}} e^{(-i / \varepsilon) S}\left(\frac{1}{i} \frac{\partial}{\partial \alpha}\left(R_{1} \psi_{n}\right)+L_{1} m_{1}+L_{2} m_{0}\right) d \alpha,
$$

which gives us $H(\varepsilon) u-\left(E+\varepsilon E_{1}\right) u=O\left(\varepsilon^{3 / 2}\right)$. By the Fredholm alternative we can choose $m_{1}$ so that (16) holds provided

$$
\int_{D}\left(L_{1} m_{0}-i \frac{\partial}{\partial \alpha}\left(R(y, \alpha) m_{0}\right)\right) \bar{\psi}_{n}\left(x, \frac{\partial S}{\partial y}+y_{1} \hat{e}_{2}\right) d x
$$

vanishes on $\{(y, \alpha):(\partial S / \partial \alpha)(y, \alpha)=0\}$, i.e. at $(y, \alpha)$ corresponding to points on $L\left(E, k_{2}, k_{3}\right)$. As in (6), the computation of Appendix 1 shows that (17) equals

$$
i \frac{\partial E_{n}}{\partial k}\left(\frac{\partial S}{\partial y}+y_{1} \hat{e}_{2}\right) \cdot \frac{\partial f}{\partial y}-i R(y, \alpha) \frac{\partial f}{\partial \alpha}+l(y, \alpha) f .
$$

On the set $(\partial S / \partial \alpha)(y, \alpha)=0$, the mapping $\psi$ from $(y, \alpha)$ to $(y, \partial S / \partial y)$ is a diffeomorphism into $L\left(E, k_{2}, k_{3}\right)$, which identifies $\alpha$ with the function $\xi_{1}$ on $L\left(E, k_{2}, k_{3}\right)$. Thus under the flow (12),

$$
\dot{\alpha}=\dot{\zeta}_{1}=-\frac{\partial E_{n}}{\partial k_{2}}\left(\xi+y_{1} \hat{e}_{2}\right)=-R(y, \alpha),
$$

when $(\partial S / \partial \alpha)(y, \alpha)=0$ and $\psi(y, \alpha)=(y, \xi)$. Thus, using $\psi$ to identify $f$ with a function on $L\left(E, k_{2}, k_{3}\right)$ when $(\partial S / \partial \alpha)(y, \alpha)=0$, the first order terms in (18) again represent the derivative of $f$ with respect to the flow in (12). Further computation (see (A.6) in Appendix 1) shows that. when one includes the lower order term $l(y, \alpha) f,(18)$ will vanish on $L\left(E, k_{2}, k_{3}\right)$ if $f=e^{-i d} g(\alpha)$, where $d s d y_{2} d y_{3}=g^{2} d \alpha d y_{2} d y_{3}$. Note that this choice makes $f$ a function of $\alpha$ alone.

To see that this choice of $u$ in (8.2) reduces to the choice of $u$ in (8.1) away from $\Sigma$, we need only expand (8.2) by stationary phase. This gives

$$
u=\left|h^{\prime \prime}(\alpha(y))\right|^{-1 / 2} \exp \left(-\frac{i}{\varepsilon} S(y, \alpha(y))-\frac{i \pi}{4} \operatorname{sgn} h^{\prime \prime}(\alpha(y))\right)\left(m_{0}(y / \varepsilon, y, \alpha(y))+0(\varepsilon)\right) \text {, }
$$

where $\alpha(y)$ is obtained by solving

$$
y_{1}+k_{2}=h^{\prime}(\alpha)
$$

using the implicit function theorem. Since (20) is precisely the equation relating the coordinates $\alpha$ and $y_{1}$ on $L\left(E, k_{2}, k_{3}\right)$, we have

$$
d y_{1} d y_{2} d y_{3}=\left|\frac{d y_{1}}{d \alpha}\right| d \alpha d y_{2} d y_{3}=\left|h^{\prime \prime}(\alpha(y))\right| d \alpha d y_{2} d y_{3},
$$

and

$$
\frac{\partial S}{\partial y}(y, \alpha(y))=\left(\alpha(y), k_{2}, k_{3}\right)=\left(f^{\prime}\left(y_{1}+k_{2}\right), k_{2}, k_{3}\right) .
$$

Hence, the principal term in (19) agrees with the order 0 term in (8.1) up to an additive constant in the phase $S$. To choose these constants consistently on 
$L\left(E, k_{2}, k_{3}\right)$, we must have $k_{i}=2 \pi m_{i} / N \alpha_{i}, i=2,3$ and as we move around $\tilde{\gamma}\left(E, k_{3}\right)$ (see (9)), the total increase in $S$ plus the net effect of the shifts $\left(i \pi \operatorname{sgn} h^{\prime \prime}(\alpha)\right) / 4$ must be an integer multiple of $2 \pi \varepsilon$. Since $\gamma\left(E, k_{3}\right)$ is a simple closed curve, the net shift is $\pi$, while the integral of $\partial S / \partial y$ around $\tilde{\gamma}$ gives the area $A\left(E, k_{3}\right)$ enclosed by $\gamma\left(E, k_{3}\right)$. Thus we must require Onsager's relation

$$
A\left(E, k_{3}\right)=2 \pi \varepsilon\left(m+\frac{1}{2}\right), \quad m \in \mathbf{Z} .
$$

Since the functions $u$ in (8.1) and (19) both satisfy $H(\varepsilon) u-\left(E+\varepsilon E_{1}\right) u=0$ to order $\varepsilon^{2}$, the order $\varepsilon$ terms in (19) must agree with those in (8.1) provided we choose the free $\psi_{n}(x) f(y, \alpha)$ in $m_{1}(x, y, \alpha)$ consistently. The reader will see that this is always possible and poses no additional constraints on $L\left(E, k_{2}, k_{3}\right)$.

Finally we piece together our local solutions using a partition of unity in $y_{1}$ such that all elements of the partition of unity are either 1 or 0 on a neighborhood of $\Sigma$. This completes the construction.

To collect the hypotheses which arose in the preceding construction and make the dependence on parameters clear. We state our result as follows:

Theorem. Assume that the equations $E_{n}(\xi)=E_{0}, \xi_{3}=k_{3}^{0}$ have a simple, closed curve $\gamma_{0}$ as one component of their solution set, and, on $\gamma_{0}, E_{n}$ is simple and

$$
\left(\frac{\partial E_{n}}{\partial k_{1}}, \frac{\partial E_{n}}{\partial k_{2}}\right)
$$

does not vanish. Then, for $\left|E-E_{0}\right| \leqq c_{1}$ and $\left|k_{3}-k_{3}^{0}\right| \leqq c_{3}, E_{n}(\xi)=E$ and $\xi_{3}=k_{3}$ define $\gamma\left(E, k_{3}\right)$ with the same properties, and we let $A\left(E, k_{3}\right)$ be the area enclosed by $\gamma\left(E, k_{3}\right)$.

For $\left|E-E_{0}\right| \leqq c_{1}$ we consider

$$
\begin{aligned}
S(N, E) & =\left\{\left(\varepsilon, k_{2}, k_{3}\right): 0<\varepsilon \leqq \varepsilon_{0} \text { and }\left|k_{3}-k_{3}^{0}\right| \leqq c_{3}\right\} \\
& \cap\left\{\left(\varepsilon, \frac{2 \pi m_{2}}{\alpha_{2} N}, \frac{2 \pi m_{3}}{\alpha_{3} N}\right): A\left(E, \frac{2 \pi m_{3}}{\alpha_{3} N}\right)=2 \pi\left(m_{1}+\frac{1}{2}\right) \varepsilon, m \in \mathbf{Z}^{3}\right\} .
\end{aligned}
$$

Then for $\left(\varepsilon, k_{2}, k_{3}\right) \in S(N, E)$ the preceding construction gives $u(y)$ satisfying

$$
\left|\left(H(\varepsilon)-\left(E+\varepsilon E_{1}\right)\right) u(y)\right| \leqq C \varepsilon^{3 / 2}, \quad y \in \mathbf{R}^{3},
$$

such that

(i) $u$ has period $N \alpha_{i}$ \& in $y_{i}, i=2,3$, and is localized in $\xi_{2}^{-}-k_{2} \leqq y_{1} \leqq \xi_{2}^{+}-k_{2}$, where $\xi_{2}^{+}$and $\xi_{2}^{-}$are the maximum and minimum of $\xi_{2}$ on $\gamma\left(E, k_{3}\right)$, and

(ii) the constant $C$ in (21) is uniform on $S(N, E)$ and independent of $N$ and $E$ for $\left|E-E_{0}\right| \leqq c_{1}$. Moreover, the integral of $|u|^{2}$ over the slab $a<y_{2}<b, c<y_{3}<d$ is bounded away from zero with the same uniformity.

Remark. In this construction the functions $m_{i}(x, y)$ and $m_{i}(x, y, \alpha), i=0,1$, in (8.1) and (8.2), respectively, are independent of $y_{2}$ and $y_{3}$. Hence, we can use exactly the same procedure to solve for the higher $m_{r}(x, y)$ and $m_{r}(x, y, \alpha)$. This will give $u$ satisfying

$$
\left|\left(H(\varepsilon)-\left(E+\varepsilon E_{1}+\cdots+\varepsilon^{M} E_{M}\right)\right) u(y)\right| \leqq C \varepsilon^{M+(1 / 2)}
$$


with the same uniformity as before. To all orders the amplitudes $m_{k}$ in $u$ will be independent of $y_{2}$ and $y_{3}$. Note that for $r \geqq 2, m_{r}(x, y)$, modulo $f_{r}(y) \psi_{n}(x, k)$, is determined by

where

$$
L_{0} m_{r}+L_{1} m_{r-1}+L_{2} m_{r-2}=E_{2} m_{r-2}+\cdots+E_{r} m_{0}
$$

$$
\begin{aligned}
& L_{0}=\left(i \frac{\partial}{\partial x}+k\right)^{2}+V(x)-E, \\
& L_{1}=-2 \frac{\partial}{\partial x} \cdot \frac{\partial}{\partial y}+2 i k \cdot \frac{\partial}{\partial y}+i \frac{\partial}{\partial y} \cdot k-E_{1}, \\
& L_{2}=-\frac{\partial}{\partial y} \cdot \frac{\partial}{\partial y} \text { and } k=k\left(y_{1}\right)=\frac{\partial S}{\partial y}+y_{1} \hat{e}_{2} .
\end{aligned}
$$

\section{Appendix I}

We need to compute

$$
I(y, k)=\int_{D} \bar{\psi}_{n}(x, k)\left(2 i k \cdot \frac{\partial}{\partial y}-2 \frac{\partial}{\partial x} \cdot \frac{\partial}{\partial y}+i \frac{\partial}{\partial y} \cdot k\right) f(y) \psi_{n}(x, k) d x,
$$

where the real vector $k$ depends on $y$. With different choices of $k$ this computation is used in formulas (6), (11) and (18).

Since $E_{n}(k)$ is a simple eigenvalue, we may assume $\psi(x, k)$ is analytic in $k$. Thus, taking the gradient with respect to $k$,

$$
\left(\left(i \frac{\partial}{\partial x}+k\right)^{2}+V(x)-E_{n}\right) \frac{\partial \psi_{n}}{\partial k}=\frac{\partial E_{n}}{\partial k} \psi_{n}-2\left(i \frac{\partial}{\partial x}+k\right) \psi_{n}
$$

and, taking the inner product with $\psi_{n}$,

$$
0=\frac{\partial E_{n}}{\partial k}(k)-2 \int_{D} \bar{\psi}_{n}(x, k)\left(i \frac{\partial}{\partial x}+k\right) \psi_{n}(x, k) d x .
$$

Expanding (A.1) gives

$$
\begin{aligned}
I(y, k)= & 2 i k \cdot \frac{\partial f}{\partial y}+2 i k f \cdot \int_{D} \bar{\psi}_{n} \frac{\partial \psi_{n}}{\partial y} d x-2 \int_{D} \bar{\psi}_{n} \frac{\partial \psi_{n}}{\partial x} d x \cdot \frac{\partial f}{\partial y} \\
& -2 f \int_{D} \bar{\psi}_{n} \frac{\partial}{\partial y} \cdot \frac{\partial \psi_{n}}{\partial x} d x+i\left(\frac{\partial}{\partial y} \cdot k\right) f .
\end{aligned}
$$

Hence, using (A.2) we have

$$
\begin{aligned}
I(y, k)= & i \frac{\partial E_{n}}{\partial k} \cdot \frac{\partial f}{\partial y}+\frac{i}{2}\left(\frac{\partial}{\partial y} \cdot \frac{\partial E_{n}}{\partial k}\right) f \\
& +f\left[-\int_{D} \bar{\psi}_{n} \frac{\partial}{\partial y} \cdot \frac{\partial \psi_{n}}{\partial x} d x+\int_{D} \frac{\partial \bar{\psi}_{n}}{\partial y} \cdot \frac{\partial \psi_{n}}{\partial x} d x+2 i k \cdot \int_{D} \bar{\psi}_{n} \frac{\partial \psi_{n}}{\partial y} d x\right], \\
I(y, k)= & i \frac{\partial E_{n}}{\partial k} \cdot \frac{\partial f}{\partial y}+\frac{i}{2}\left(\frac{\partial}{\partial y} \cdot \frac{\partial E_{n}}{\partial k}\right) f+c f,
\end{aligned}
$$


where

$$
\begin{aligned}
& c=\int\left[\frac{\partial \bar{\psi}_{n}}{\partial x} \frac{\partial \psi_{n}}{\partial y}+\frac{\partial \bar{\psi}_{n}}{\partial y} \cdot \frac{\partial \psi_{n}}{\partial x}+2 i k \cdot \bar{\psi} \frac{\partial \psi_{n}}{\partial y}-i k \cdot \frac{\partial \bar{\psi}_{n}}{\partial y} \psi_{n}\right] d x, \\
& c=2 \operatorname{Re}\left\{\int_{D} \frac{\partial \bar{\psi}_{n}}{\partial y} \cdot\left(\frac{\partial}{\partial x}-i k\right) \psi_{n} d x\right\}, \\
& c=\operatorname{Re}\left\{i \int_{D} \frac{\partial \bar{\psi}_{n}}{\partial y} \cdot\left(\left(H_{0}-E_{n}\right) \frac{\partial \psi_{n}}{\partial k}-\frac{\partial E_{n}}{\partial k} \psi_{n}\right) d x\right\},
\end{aligned}
$$

where $H_{0}=(i(\partial / \partial x)+k)^{2}+V(x)$. Since $H_{0}$ is self-adjoint,

$$
\int_{D} \frac{\partial \bar{\psi}_{n}}{\partial k_{i}}\left(H_{0}-E_{n}\right) \frac{\partial \psi}{\partial k_{j}} d x=\int_{D} \frac{\partial \bar{\psi}_{n}}{\partial k_{j}}\left(H_{0}-E\right) \frac{\partial \psi_{n}}{\partial k_{i}} d x .
$$

Moreover, the matrix $M$ given by $(M)_{i j}=\partial k_{i} / \partial y_{j}$ satisfies

$$
M \frac{\partial E_{n}}{\partial k}=\frac{\partial E_{n}(k)}{\partial y}+\left(M-M^{t}\right) \frac{\partial E_{n}}{\partial k} .
$$

Thus, letting $A$ denote the anti-symmetric part of $M$, (A.3) becomes

$$
\begin{aligned}
& c=i \int_{D} \frac{\partial \bar{\psi}_{n}}{\partial k} \cdot A\left(\left(H_{0}-E_{n}\right) \frac{\partial \psi_{n}}{\partial k}-\frac{\partial E_{n}}{\partial k} \psi_{n}\right) d x-\operatorname{Re}\left\{i \int_{D} \frac{\partial \bar{\psi}_{n}}{\partial k} \cdot \frac{\partial E_{n}}{\partial y} \psi_{n} d x\right\}, \\
& c=-2 i \int_{D} \frac{\partial \bar{\psi}_{n}}{\partial k} \cdot A\left(i \frac{\partial}{\partial x}+k\right) \psi_{n} d x-\operatorname{Re}\left\{i \int_{D} \frac{\partial \bar{\psi}_{n}}{\partial k} \cdot \frac{\partial E_{n}}{\partial y} \psi_{n} d x\right\} .
\end{aligned}
$$

In (5) we have $\partial E_{n} / \partial y=\partial^{2} \varphi / \partial y \partial s=\partial k / \partial s$ so that (A.4) becomes

$$
c=-2 i \int_{D} \frac{\partial \bar{\psi}_{n}}{\partial k} \cdot A\left(i \frac{\partial}{\partial x}+k\right) \psi_{n} d x+i \int_{D} \bar{\psi}_{n} \frac{\partial \psi_{n}}{\partial s} d x
$$

and (5) becomes

$$
0=\int_{D} \bar{\psi}_{n} L_{1} m_{0} d x=i \frac{\partial f_{0}}{\partial s}+i f_{0} \int_{D} \bar{\psi}_{n} \frac{\partial \psi_{n}}{\partial s} d x-i \frac{\partial E_{n}}{\partial k} \cdot \frac{\partial f_{0}}{\partial y}-\frac{i}{2}\left(\frac{\partial}{\partial y} \cdot \frac{\partial E}{\partial k}\right) f_{0}-c f_{0},
$$

which immediately gives (6).

In (11) $\partial E_{n} / \partial y=0$, so that (A.4) becomes

$$
c=-2 i \int_{D} \frac{\partial \bar{\psi}_{n}}{\partial k} \cdot A\left(i \frac{\partial}{\partial x}+k\right) \psi_{n} d x
$$

and $b$ in (11) satisfies

$$
b=-c=2 i \int_{D} \frac{\partial \bar{\psi}_{n}}{\partial k} \cdot A\left(i \frac{\partial}{\partial x}+k\right) \psi_{n} d x
$$

as claimed.

Finally, when we use (A.4), the expression in (17) becomes

$$
i \frac{\partial E_{n}}{\partial k} \cdot \frac{\partial f}{\partial y}+\frac{i}{2}\left(\frac{\partial}{\partial y} \cdot \frac{\partial E_{n}}{\partial k}\right) f+\left(c-E_{1}\right) f-i R(y, \alpha) \frac{\partial f}{\partial \alpha}-i \frac{\partial R}{\partial \alpha} f-i R f \int_{D} \bar{\psi}_{n} \frac{\partial \psi_{n}}{\partial \alpha} d x .
$$


This establishes (18) with

$$
l(y, \alpha)=\frac{i}{2} \frac{\partial}{\partial y} \cdot \frac{\partial E_{n}}{\partial k}+c-E_{1}-i \frac{\partial R}{\partial \alpha}-i R \int_{D} \bar{\psi}_{n} \frac{\partial \psi_{n}}{\partial \alpha} d x
$$

but we still need to study the restriction of $l$ to $L\left(E, k_{2}, k_{3}\right)$.

From the definition of $R$

$$
E\left(\alpha, k_{2}+y \hat{e}_{2}, k_{3}\right)-E=\left(y_{1}+k_{2}-h^{\prime}(\alpha)\right) R,
$$

and on $L\left(E, k_{2}, k_{3}\right), y_{1}+k_{2}=h^{\prime}(\alpha)$. Hence on $L\left(E, k_{2}, k_{3}\right), R=\partial E_{n} / \partial k_{2}\left(\alpha, h^{\prime}(\alpha), k_{3}\right)$ and $\partial R / \partial y_{1}=1 / 2\left(\partial^{2} E_{n}\right) / \partial k_{2}^{2}\left(\alpha, h^{\prime}(\alpha), k_{3}\right)$. Thus, differentiating the identity

$$
R\left(h^{\prime}(\alpha)-k_{2}, \alpha\right)=\frac{\partial E_{n}}{\partial k_{2}}\left(\alpha, h^{\prime}(\alpha), k_{3}\right)
$$

with respect to $\alpha$, we conclude that

$$
\frac{\partial R}{\partial \alpha}\left(h^{\prime}(\alpha)-k_{2}, \alpha\right)=\frac{\partial^{2} E_{n}}{\partial k_{1} \partial k_{2}}\left(\alpha, h^{\prime}(\alpha), k_{3}\right)+\frac{h^{\prime \prime}(\alpha)}{2} \frac{\partial^{2} E_{n}}{\partial k_{2}^{2}}\left(\alpha, h^{\prime}(\alpha), k_{3}\right) .
$$

Thus on $L\left(E, k_{2}, k_{3}\right),(\mathrm{A} .5)$ becomes

$$
l(y, \alpha)=\frac{i}{2} \frac{\partial}{\partial \alpha}\left(-\frac{\partial E_{n}}{\partial k_{2}}\left(\alpha, h^{\prime}(\alpha), k_{3}\right)\right)-2 i \int_{D_{n}} \frac{\partial \bar{\psi}_{n}}{\partial k} \cdot A\left(i \frac{\partial}{\partial x}+k\right) \psi_{n} d x-E_{1},
$$

so that (17), on $L\left(E, k_{2}, k_{3}\right)$ coordinatized by $\left(y^{\prime}, \alpha\right)=\left(y_{2}, y_{3}, \alpha\right)$, is simply

$$
i v\left(y^{\prime}, \alpha\right) \cdot\left(\frac{\partial f}{\partial y^{\prime}}, \frac{\partial f}{\partial \alpha}\right)+\frac{i}{2}\left(\left(\frac{\partial}{\partial y^{\prime}}, \frac{\partial}{\partial \alpha}\right) \cdot v\right) f-\left(b+E_{1}\right) f
$$

where

$$
v=\left.\left(\frac{\partial E_{n}}{\partial k_{2}}, \frac{\partial E_{n}}{\partial k_{3}},-\frac{\partial E_{n}}{\partial k_{2}}\right)\right|_{k=\left(\alpha, h^{\prime}(\alpha), k_{3}\right)}
$$

Since the flow in (12) in these coordinates has $\dot{\alpha}=-\partial E / \partial k_{2}$, this is the desired result.

Acknowledgements. The results presented here were described in [5] and we wish to thank the organizers of the VIII Escola Latino-Americana de Mathematica for their support. We also wish to thank Walter Kohn for a helpful discussion of these problems, and to thank J. Avron for helpful comments on an earlier version of this paper.

\section{References}

1. Ashcroft, N., Mermin, N. D.: Solid state physics. Philadelphia, PA: Holt, Rinehart and Winston 1976

2. Benssousan, A., Lions, J. L., Papanicolaou, G.: Asymptotic analysis for periodic structures. Amsterdam: North-Holland 1978

3. Chambers, R. G.: The wave function of a Bloch electron in a magnetic field. Proc. Phys. Soc. 89, 695-710 (1966)

4. Duistermaat, J. J.: Oscillatory integrals, Lagrange immersions and unfolding of singularities. Commun. Pure Appl. Math. 27, 207-281 (1974)

5. Guillot, J., Ralston, J., Trubowitz, E.: Semi-classical approximations in solid state physics. To appear in proceedings VIII ELAM, Rio de Janeiro, July 1986 
6. Helffer, B.: Opérateurs de Schrödinger avec champ magnetıque. Seminaire Equations aux Derivées Partielles, 1986-1987, Expose' X

7. Helffer, B., Sjöstrand, J.: Effect tunnel pour l'equation de Schrödinger avec champ magnetique. Preprint December 1986

8. Keller, J.: Corrected Bohr-Sommerfeld quantum conditions for nonseparable systems. Ann. Phys. 4, 180-188 (1958)

9. Keller, J., Rubinow, S.: Asymptotic solution of eigenvalue problems. Ann. Phys. 9, 24-75 (1960)

10. Kohn, W.: Theory of Bloch electrons in a magnetic field: The effective Hamiltonians. Phys. Rev. 115, 1460-1478 (1959)

11. Maslov, V. P., Fedoriuk, M. V.: Semi-classical approximation in quantum mechanics. Dordrecht: D. Reidel 1981

12. Onsager, L.: Interpretation of the de Haas-van Alphen effect. Phil. Mag. 43, 1006-1008 (1952)

13. Peierls, R.: Zur Theorie des Diamagnetismus von Leitungselektronen. Z. Phys. 80, 763-791 (1933)

14. Zak, J.: Dynamics of electrons in solids in external fields. Phys. Rev. 168, 686-695 (1968)

Communicated by A. Jaffe

Received August 31, 1987; in revised form October 26, 1987 
